\title{
OBITUARI: LAMBERT FERRERES (1952-2020)
}

\author{
XAVIER ESPLUGA \\ Universitat de Barcelona \\ xespluga@ub.edu \\ ORCID: 0000-0002-6690-1974
}

El primer dia del mes més cruel de l'any, en aquest tràgic 2020, moria a Barcelona el professor Lambert Ferreres, Catedràtic de Filologia Llatina de la Universitat de Barcelona.

Llicenciat (1974) i doctorat (1977) en Filosofia i Lletres (en l'especialitat de Filologia Clàssica) per la mateixa institució, Ferreres es va incorporar ben aviat com a docent a l'aleshores Departament de Filologia Clàssica i, amb el pas del temps, va recórrer les diverses etapes del cursus honorum acadèmic: ajudant (1974-1976), professor adjunt (1977-1983) i titular de Filologia Llatina (1983-1993). La carrera a la Universitat de Barcelona només va patir una breu interrupció en els anys 90, quan es va incorporar a la Universitat Autònoma de Barcelona (UAB) en haver-hi guanyat la càtedra de Filologia Llatina. Al campus de Bellaterra hi restà tres cursos acadèmics (1993-1995) fins que en aquest darrer any de 1995 tornà a l'alma mater barcelonina.

Durant tots aquests anys, el doctor Ferreres es va implicar activament en la docència universitària i en la gestió acadèmica. Va impartir cursos d'especialització -ara de màster, abans de doctorat- sobre la seva principal competència científica: el llatí i els textos dels cristians. En aquest àmbit acadèmic va ser tutor de diversos treballs $\mathrm{i}$ responsable acadèmic del programa de doctorat que -amb noms diversos i juntament $\mathrm{amb}$ altres àrees- s'impartia des de l'àrea de Filologia Llatina. A la llicenciatura -ara, grau- també es va encarregar d'assignatures de "textos", d'autors fonamentals de la literatura llatina (Ciceró, Livi, Virgili), sense renunciar a assumir els cursos propedèutics de llatí, aquells que donen la benvinguda en el primer any als nous estudiants de Filologia Clàssica, perquè estava fermament convençut de la importància d'aquesta formació inicial.

Fou membre del grup d'innovació docent Hesperia Latina (2007-2020) i del grup de recerca Littera i director del Departament de Filologia Llatina durant set anys (19972004). Va ser vocal de la junta directiva de la Secció Catalana de la Sociedad Española de Estudios Clásicos (SEEC) en diverses ocasions fins arribar a ser-ne vicepresident i tresorer (2008-2012). Des d'aquesta instància, va colllaborar en l'organització d'alguns dels famosos simposis, dels quals era participant assidu. Fou el responsable de l'edició de les actes del IXè simposi -el celebrat a Sant Feliu de Guíxols l'any 1988- publicades l'any 1991, ${ }^{1}$ que es dedicaren al mestre Bejarano coincidint amb la seva jubilació; també fou el coeditor -juntament amb Esperança Borrell- del segon volum de les actes del XVIè simposi celebrat a Tarragona el 2009, aparegut l'any 2010.

\footnotetext{
${ }^{1}$ Ferreres (1991C).
} 
Més enllà d'aquestes servituds acadèmiques, emperò, Ferreres serà recordat per haver conreat, amb devoció, una parcel-la exigent de la Filologia Llatina: els textos cristians i les seves particularitats lingüístiques. En efecte, en els anys 70 i sota l'ègida del doctor Virgilio Bejarano, que aleshores ocupava de la primera càtedra de Filologia Llatina de la Universitat de Barcelona, Ferreres es va endinsar en el que, amb el pas del temps, seria el seu principal camp d'especialització. Per entendre el context en què es va produir l'experiència formativa de Lambert Ferreres i de bona part dels seus condeixebles és molt recomanable llegir-ne l'aprofundida notícia que el mateix Ferreres va publicar als Cuadernos de Filología Clásica del 2008, on glossava sentidament la figura del mestre, ${ }^{2}$ la veu que li dedica al volum col-lectiu La nissaga catalana (2011), ${ }^{3}$ i més encara l'evocació que en feu l'any 2011 en ocasió del congrés sobre llatí medieval ibèric. ${ }^{4}$ En efecte, des de la seva incorporació a la Universitat de Barcelona, procedent d'Uppsala, Bejarano havia cultivat amb especial atenció la llatinitat cristiana, tot publicant i dirigint treballs acadèmics -memòries de llicenciatura i tesis doctorals-, en un esforç d'especialització que alguns dels seus deixebles -en particular, Jordi Avilés, Esperança Borrell i el mateix Ferreres- continuarien.

Precisament, en els primers estadis de llur activitat formativa les trajectòries del doctor Ferreres i del doctor Avilés concorregueren en paral-lel: les memòries de llicenciatura de tots dos, presentades l'any 1974, estaven dedicades a l'estudi de les versions bíbliques anteriors a la Vulgata, en particular les versions del Llibre de la Saviesa citades per autors hispans i africans: mentre el doctor Avilés s'encarregava dels capítols 1-3 del Llibre de la saviesa, ${ }^{5}$ el doctor Ferreres feia el mateix amb els capítols 4-6. ${ }^{6}$ Poc després, l'any 1976, tots dos signaren un primer article -el primer de llur carrera- sobre la tradició indirecta del Llibre de la Saviesa publicat en el segon número de la present revista - $\mathrm{l}^{\prime}$ Anuari de Filologia- on Ferreres col-laborà en tantes ocasions. ${ }^{7}$

També van anar en paral-lel les recerques que conduirien a llurs tesis doctorals, presentades i defensades l'any 1977. Tots dos, Avilés i Ferreres, se centraren en l'obra de Lucifer de Càller, bisbe sard del segle IV, polèmic i rigorista, opositor de la política religiosa de Constanci II (377-361), que acabà essent enviat a l'exili pel mateix emperador. Ambdues tesis compartien el mateix títol principal: Studia Luciferiana. Mentre Avilés -amb Studia Luciferiana I- s'havia centrat en el tractat De regibus apostaticis del bisbe sard, ${ }^{8}$ Ferreres -amb Studia Luciferiana II- havia escollit el Moriundum esse pro Dei filio, escrit per Lucifer cap al $361 .{ }^{9}$ Es tractava d'un treball de filologia formal que

\footnotetext{
2 Ferreres (2008).

${ }^{3}$ Ferreres (2011B).

${ }^{4}$ Ferreres (2011). En aquest mateix volum, s'hi pot llegir el record d'un altre del doctor Mayer, un altre dels deixebles de Bejarano. Vegeu Mayer (2011).

5 Avilés (1974).

${ }^{6}$ Ferreres (1974).

${ }^{7}$ Avilés, Ferreres (1976).

${ }^{8}$ Avilés (1977). Un estudi i l'edició crítica del text foren publicats a Analecta Sacra Tarraconensia corresponent al 1976/1977. Vegeu Avilés (1976-1977).

9 Ferreres (1977). Una part de la tesi, amb l'edició crítica del text, fou publicat a Analecta Sacra Tarraconensia del 1980-1981. Vegeu Ferreres (1980-1981).
} 
comportà l'edició crítica del text, amb la col·lació dels dos testimonis manuscrits, l'estudi i un index uerborum, realitzat aleshores a partir de fitxes manuals, seguint les sàvies recomanacions del doctor Bejarano. També tots dos -Avilés i Ferreres- presentaren conjuntament una comunicació sobre la figura del controvertit bisbe de Càller al Vè congrés espanyol d'estudis clàssics celebrat a Madrid el $1978,{ }^{10}$ on abordaven els elements de retòrica en la prosa de l'enèrgic bisbe sard. A finals dels anys 80 , les recerques luciferianes dutes a terme a Barcelona coincidirien amb la publicació de dues edicions foranes, senyal de l'interès renascut per la figura del polemista callerità: l'any 1978 aparegué el volum corresponent del Corpus Christianorum Series Latina (CCSL 8) dedicat precisament a l'opera omnia del bisbe sard, en edició del llatinista neerlandès Gerardus Frederik Diercks, ${ }^{11}$ que incorporava tant el Moriundum esse pro Dei filio (CPL 116) que havia editat Ferreres com el De regibus apostaticis (CPL 113) que havia estudiat Avilés; poc després, l'any 1980, apareixia a Lecce l'edició, amb traducció italiana, d'aquests dos mateixos tractats a càrrec del professor Valerio Ugenti, que amb el pas del temps esdevindria bon amic del doctor Ferreres. ${ }^{12}$ En qualsevol cas, Ferreres, que amb anterioritat ja havia presentat un estudi sobre les possibles fonts de Lucifer, ${ }^{13}$ acabaria publicant la seva pròpia edició del Moriundum esse pro Dei filio a la revista Analecta Sacra Tarraconensia en el número corresponent al 1980/1981. ${ }^{14}$ També publicà les concordances de l'opera omnia luciferiana (1987) ${ }^{15}$ i aprofundí tant en l'estudi de les fonts, en particular rastrejant-hi la presència de Ciceró (1992), ${ }^{16}$ com en la recepció successiva de l'obra luciferiana en la figura de Vicenç de Lerins (1992). ${ }^{17}$

Tot i que paulatinament la figura del bisbe de Càller cedia davant l'aparició de noves ocupacions científiques, el text de Lucifer encara generaria interès en temps més recents: l'any 1992 Ferreres publicava una breu nota sobre la relació entre els dos únics testimonis manuscrits de l'obra de Lucifer -el ms. 1351 de la Biblioteca Pública i Universitària de Ginebra (G) i el ms. Reg. Lat. 133 de la Biblioteca Apostòlica Vaticana (V)- en la qual reexaminava la proposta de Diercks de fer dependre $\mathbf{G}$ de $\mathbf{V}$, a través de l'exemplar uetustissimum perdut posseït per l'abadia de Corbie. ${ }^{18}$ L'any 1996 Ferreres va participar en el congrés celebrat a Càller i dedicat íntegrament a la figura del polèmic bisbe amb una comunicació sobre les oracions completives en la prosa luciferiana; ${ }^{19}$ en aquella ocasió va conèixer Sonia Laconi, professora de Llengua i Literatura Llatina de la Universitat de Càller, organitzadora de l'esdeveniment, que havia estudiat el text del

\footnotetext{
10 Avilés, Ferreres (1978).

11 Diercks (1978).

12 Ugenti (1980).

13 Ferreres (1977B).

14 Ferreres (1980-1981).

${ }^{15}$ Ferreres (1987).

16 Ferreres (1992E).

17 Ferreres (1992C).

18 Ferreres (1992B).

${ }^{19}$ Ferreres (2001).
} 
Moriundum esse pro Dei filio, ${ }^{20}$ i n'havia fet també una edició crítica. ${ }^{21}$ De l'estudiosa sarda, interessada també en l'obra del prelat local, ${ }^{22}$ Ferreres prologà la introducció a la biografia de Costanci II, publicada el $2004 \mathrm{amb}$ el títol de Costanzo II. Ritratto di un imperatore eretico. En canvi, no em resulta que finalment publiqués el seu estudi sobre el text bíblic citat per Lucifer que havia emprès poc abans.

Després del bisbe de Càller, tocà el torn d'un altre problemàtic text de la llatinitat cristiana: em refereixo al De laude martyrii atribuït a Cebrià, bisbe de Cartago. Es tracta d'un opuscle escrit probablement vers 252-253 per algú actiu en l'obra del gran prelat africà i que, en conseqüència, ha estat transmesa amb bona part de l'obra cebrianenca, ${ }^{23}$ escrit en coincidència amb alguns grans desastres del moment, com ara la persecució de l'emperador Deci del 250 o la gran plaga que va esclatar el 249. D'aquest escrit apòcrif cebrianenc Ferreres publicà les concordances, ${ }^{24}$ n'estudià les fonts clàssiques, ${ }^{25}$ la tradició indirecta, ${ }^{26}$ i la transmissió manuscrita, la qual -segons confessió pròpiasumava centenars de manuscrits. L'interès per aquesta obra i la connexió amb Ugenti motivaren la seva participació en el congrés internacional sobre la literatura "polèmica" cristiana dels segles III i IV, celebrat a Lecce l'any 2010, les actes del qual foren publicades a la sèrie Recherches sur les rhétorique religieuses de Brepols, per Alessandro Capponi, un dels deixebles d'Ugenti i organitzador d'aquesta reunió internacional. ${ }^{27}$

Ferreres també tingué temps per ocupar-se d'altres autors de la llatinitat cristiana. Així, el 1996 col-laborà en la organització del congrés internacional sobre Pacià de Barcelona, celebrat a cavall entre Barcelona i Lió, on presentà una comunicació posteriorment publicada a les mateixes actes- sobre el text bíblic que aquest bisbe de Barcelona cita en les seves obres. ${ }^{28} \mathrm{~S}^{\prime}$ interessà per un passatge de la Història eclesiàstica de Beda (relatiu a les vicissituds del monjo Dryhthtelm) conservat en un manuscrit miscel-lani de la biblioteca de la Universitat de Barcelona (en concret, el manuscrit 231, que prové del convent de Sant Josep), ${ }^{29}$ i per les citacions augustinianes d'Elipand de Toledo. ${ }^{30}$ En els darrers temps havia estat ocupat en l'estudi de dos textos cristians particulars: d'una banda, una Glossa in Apocalypsin, ${ }^{31}$ obra datada entre el 750 i el 900, conservada en un manuscrit de la biblioteca de la Universitat de Cambridge -en concret,

${ }^{20}$ Laconi (1997).

${ }^{21}$ Laconi (1998).

22 Vegeu Laconi (1992); Laconi (2001B).

23 Vegeu-ne la recent edició, dins el Corpus Christianorum, de Ciccolini, Mattei (2016).

${ }^{24}$ Ferreres (1999A).

${ }_{25}$ Ferreres (1999B).

${ }^{26}$ Ferreres (1999C).

27 Ferreres (2012).

${ }^{28}$ Ferreres (2004).

${ }^{29}$ Ferreres (2014C).

30 Ferreres (1991B); Ferreres (1994A). L'obra del prelat visigot constituí l'objecte de la tesina de llicenciatura de José Fernández González, dirigida pel doctor Ferreres i presentada el 1992. Vegeu Fernández González (1992).

${ }^{31}$ Ferreres (2015A); Ferreres (2015B) 
el ms. Db. X.16- recentment editada per Roger Gryson al Corpus Christianorum; ${ }^{32}$ de l'altra, la versió llatina del $\Lambda o ́ \gamma o \varsigma \pi \varepsilon \rho \grave{\alpha} \sigma \kappa \kappa \eta ́ \sigma \varepsilon \omega \varsigma$, atribuït a Basili de Cesarea, ${ }^{33} \mathrm{de}$ la qual l'any passat (2019) publicava dos articles a la Revista de Estudios Latinos i a Musaeum Helveticum: aquests dos articles semblen ser les seves darreres contribucions científiques.

Plenament conscient del paper de la literatura llatina com a receptacle de les influències dels autors d'època clàssica, Ferreres va poder rastrejar la presència dels darrers entre autors cristians -Lactanci, ${ }^{34}$ Jeroni i Agustí ${ }^{35}$, , tot tenint present la importància de la tradició indirecta i de la paràfrasi, com a forma velada d'al·lusió, entre els procediments compositius dels autors de la llatinitat tardana.

No es deixi enganyar el lector, tanmateix, per aquesta especialització. La competència i solidesa de Ferreres li van permetre dedicar-se a estudis puntuals d'autors no menors d'època clàssica, analitzant-ne passatges compromesos per la transmissió textual o significatius en la seva recepció posterior: el sintagma prepositiu in suspicionem a Corneli Nepot: ${ }^{36}$ dos passatges de la quarta Catilinària; ${ }^{37}$ les citacions del De oratore ciceronià a la Institutio oratoria de Quintilià; 38 el non temptaris del vers 389 del primer llibre de l'Ars amatoria d'Ovidi; ${ }^{39}$ la variant in Palatio [monte] Lupercal del primer llibre de Livi. ${ }^{40} \mathrm{D}^{\prime}$ aquest darrer autor precisament estudià la traducció catalana anònima conservada en el ms. Harley 4893 de la British Library, contrastant-la amb el manuscrit 77 de la Biblioteca Pública i Universitària de Ginebra, que conté la versió francesa del benedictí Pierre Bersuire (del 1354-1358); ${ }^{41}$ al mateix temps va analitzar, per primera vegada, la denominada "declaració dels mots" que acompanya la tal traducció. ${ }^{42}$ Tot i que en temps més recents, Montserrat Ferrer hi ha dedicat la seva tesi doctoral (amb l'edició de la dita "declaració" en apèndix), ${ }^{43}$ no deixa de sorprendre l'interès primerenc de Ferreres per aquesta traducció nostrada.

Totes aquestes contribucions científiques revelen el profund coneixement del sistema de la llengua llatina, en les seves vessants sincròniques i diacròniques, i una especial atenció als processos i les característiques de la Sondersprache dels primers cristians llatins, com ara són els hel-lenismes o les traduccions llatines de conceptes i termes del cristianisme grec. Tal facultat li permetia moure's amb facilitat a cavall entre les particularitats lingüístiques del llatí d'època clàssica i el llatí d'època cristiana, intentant veure els canvis de significat, funció o construcció que determinats vocables

\footnotetext{
${ }^{32}$ Gryson (2013).

33 Ferreres (2014B).

${ }^{34}$ Ferreres (1995).

35 Ferreres (1985).

${ }^{36}$ Ferreres (2018A).

37 Ferreres (1992D).

38 Ferreres (1994B).

${ }^{39}$ Ferreres (1978).

${ }^{40}$ Ferreres (1989).

${ }^{41}$ Ferreres (1991A).

42 Ferreres (1991D).

${ }^{43}$ Ferrer Santanach (2011).
} 
(arentia; ${ }^{44}$ decanto; ${ }^{45}$ inanimare i interceptus; ${ }^{46}$ itaque/ita; ${ }^{47}$ praesentia ${ }^{48}$ ) adquirien entre els autors de la llatinitat cristiana.

L'activitat filològica del doctor Ferreres s'inscriu, doncs, plenament en els paràmetres de l'anomenada "Escola de Filologia de Barcelona”. Potser tenia raó Pere Quetglas quan en el seu moment la va definir com a "entidad etérea"; 49 certament ho ha pogut ser en termes institucionals, però, tanmateix 1' "Escola de Filologia de Barcelona" no deixa de ser quelcom real i tangible, un ideal que viu i traspua en l'activitat docent $i$ investigadora de moltes de les diverses persones que fan cap en l'àrea de Filologia Llatina de la Universitat de Barcelona i que es transmet, si es vol per osmosi, de generació en generació. ${ }^{50}$ En el cas específic de Lambert Ferreres, en la seva trajectòria acadèmica es van poder entrecreuar un sòlid coneixement de la llengua llatina en totes les seves variants -diacròniques, diatòpiques i diastràtiques-, un domini profund de les tècniques i instruments de la nostra disciplina -en particular, de l'ecdòtica-, i una atenció exigent als mecanismes de formació, circulació i recepció dels textos: en fi, tot aquell conjunt de coneixements, procediments i tècniques que ja el doctor Bejarano va enumerar en un conegut article publicat a la revista Durius d'ara fa bastant temps, en el qual definia, singularitzant objectius i mètodes, el recorregut de la Filologia Llatina, ${ }^{51}$ en una mena de declaració conscient de manifest programàtic sobre l'essència de la nostra disciplina. Contemplat, doncs, en perspectiva històrica -l'estrictament necessària per intentar amb gran cuita fer-ne una semblança modesta-, l'activitat docent i investigadora del doctor Ferreres s'ha inserit plenament en aquests paràmetres i ho ha fet amb la major de les acríbies filològiques, amb pulcritud i respecte pel detall.

Ara com ara, amb la seva desaparició, ha quedat certament òrfena una parcel·la els textos i la llengua dels cristians- per a la qual no s'entreveu, si més no a curt termini, la continuïtat necessària. Però, amb aquesta pèrdua prematura, inesperada i dura també queda molt tocat tot l'edifici de la llatinitat barcelonina, bastit amb l'esforç rigorós, tenaç, constant i discret de filòlegs com Lambert Ferrreres. ${ }^{52}$

${ }^{44}$ Ferreres (2014A).

${ }^{45}$ Ferreres (2019A)

${ }^{46}$ Ferreres (1992A).

${ }^{47}$ Ferreres (1979); Ferreres (1981).

${ }^{48}$ Ferreres (2019C).

${ }^{49}$ Quetglas (1981), vi. Dret a llei, cal recordar que el doctor Quetglas afegia, immediatament, rere "entidad etérea", "pero sin duda existente".

${ }^{50}$ Com a exemple de la pertinença de Ferreres a aquesta tradició, esmento la seva participació, juntament amb Pere Quetglas i Priscila Borrell, en la reedició de la Sintaxis llatina de Bassols, el pal de paller de la llatinitat barcelonina. Vegeu Bassols (2015).

${ }^{51}$ Bejarano (1975).

${ }^{52}$ Voldria donar les gràcies als companys de secció, Esperança Borrell, Jaume Juan i Noemí Moncunill, que han volgut llegir-se aquestes línies i enriquir-les amb les seves observacions. 


\section{BIBLIOGRAFIA}

AVILÉS, Jordi (1974). Versiones prejeronimianas del Libro de la sabiduría : capítulos 1-3 en los autores hispanos y africanos. Memoria de licenciatura que presenta Jorge Avilés Zapater. Dirigida por Virgilio Bejarano, Barcelona, Universitat de Barcelona.

AvILÉS, Jordi (1977). Studia luciferiana I. Estudio crítico, edición e index uerborum del tratado De regibus apostaticis. Memoria [...] para la obtención del Grado de Doctor que presenta Jorge Avilés Zapater. Elaborada bajo la dirección de Virgilio Bejarano Sánchez, Barcelona, Universitat de Barcelona, 1977.

AvILÉS, Jordi (1976-1977). “El tratado 'De regibus apostaticis' de Lucifer de Cagliari (estudio crítico y edición)", Analecta Sacra Tarraconensia 49-50, 1976-1977, 345-437.

AVILÉS, Jordi, FERRERES, Lambert (1976): “Apostillas a la tradición indirecta del Libro de la Sabiduría", Anuari de Filologia 2, 1976, p. 25-35.

AVILÉS, Jordi, FERRERES, Lambert (1978): “Algunos elementos de retórica en Lucifer de Cagliari", Actas del V Congreso Español de Estudios Clásicos, Madrid 1978, p. 331336.

BAssols De Climent, Marià (2015). Sintaxis latina. Edición de L. Ferreres, P. Quetglas y P. Borrell, Barcelona, Publicacions i Edicions de la Universitat de Barcelona, 2015.

BEjARANO, Virgilio (1975). "La filología latina: objetivos y métodos", Durius 3/1, 1975, p. 53-144.

BORRELL, Esperança, FERRERES, Lambert, eds. (2010). Artes ad humanitatem, vol. II, Barcelona, Universitat de Barcelona, 2010.

CicCOLINI, Laetitia, MATTEI, Paul (2016). Sancti Cypriani episcopi De habitu virginum cura et studio Laetitia Ciccolini. Opera Pseudo-Cyprianea. De laude martyrii; Ad Vigilium episcopum de Iudaica incredulitate; De re baptismate cura et studio Laetitia Ciccolini et Paul Mattei, Turnout, Brepols, 2016 (Corpus Christianorum. Series Latina, 28).

DIERCKS, Gerardus Frederik (1978). Lucifer Calaritanus. Opera quae supersunt, Turnhout, Brepols, 1978 (Corpus Christianorum Series Latina 8).

FERNÁNDEZ GONZÁLEZ, José (1992). Index uerborum Elipandi. Memoria que que presenta José Fernández González para la obtención del grado de licenciado, bajo la dirección del Dr. Lambert Ferreres Pérez, Barcelona, Universitat de Barcelona, 1992.

FERRER SANTANACH, Montserrat (2011). La traducció catalana medieval de les Dècades de Titus Livi : edició del llibre I. Tesi doctoral dirigida pel Dr. Josep Pujol Gómez, Bellaterra, Universitat Autònoma de Barcelona, 2011.

FERRERES, Lambert (1974). Versiones prejeronimianas del Libro de la sabiduría : capítulos 4-6 en los autores hispanos y africanos. Memoria de licenciatura que presenta Lamberto Ferreres Pérez. Dirigida por Virgilio Bejarano Sánchez, Barcelona, Universitat de Barcelona.

FERRERES, Lambert (1977A). Studia luciferiana II : estudio crítico, edición e Index uerborum del tratatado Moriundum esse pro dei filio. Memoria elaborada [...] que para la obtención del Grado de Doctor presenta Lamberto Ferreres Pérez bajo la dirección de Virgilio Bejarano Sánchez, Barcelona, Universitat de Barcelona, 1977.

FERRERES, Lambert (1977B). “Las fuentes de Lucifer de Cálaris en su 'Moriundum esse pro dei filio"', Anuari de Filologia 3, 1977, p. 101-115. 
FERRERES, Lambert (1978). “¿Non temptaris? Ars amatoria, I, 389”, Helmántica 88/29, 1978, p. 61-64

FERRERES, Lambert (1979). "Itaque en lloc de ita en llatí tardà", Faventia 1/2, 1979, p. 207-210

FERRERES, Lambert (1980-1981). “El tratado 'Moriundum esse pro Dei Filio' de Lucifer de Cagliari”, Analecta Sacra Tarraconensia 53-54, 1980-1981, 1-99.

FERRERES, Lambert (1981): “De itaque pro ita usu”, Emerita 49/1, 1981, p. 59-60.

FERRERES, Lambert (1985). "Presencia de Virgili a Lactanci", Studia Virgiliana. Actes del VIè Simposi d'Estudis Clàssics, 11-13 de febrer de 1981, Bellaterra, Universitat Autònoma de Barcelona, 1985 (Estudis de literatura comparada, 1), p. 147-152.

FERRERES, Lambert (1987). Index uerborum Luciferi Calaritani (Mor.), Barcelona, Universitat de Barcelona, 1987.

FERRERES, Lambert (1989). “Lupercal: nota a Livio 1, 5, 1”, Faventia 11/2, 1989, p. $157-162$

FERRERES, Lambert (1991A). “El ms. fr. 77 de la B.P.U. de Ginebra i la traducció catalana anònima de Titus Livi", Annals de l'Institut d'Estudis Gironins 31, 1991, p. 23-29.

FERRERES, Lambert (1991B). “A propósito de dos supestas citas augustinianas en Elipando", en L. FERRERES, ed. (1991), p. 771-774.

FERRERES, Lambert, ed. (1991C). Actes del IXè simposi de la secció catalana de la SEEC : St. Feliu de Guíxols : 13-16 d'abril de 1988 : treballs en honor de Virgilio Bejarano, Barcelona, Publicacions de la Universitat de Barcelona, 1991 (Aurea Saecula 1-2).

FERRERES, Lambert (1991D). "La 'declaració dels mots' de la traducció catalana anònima de Titus Livi", Anuari de Filologia 14/2, 1991, p. 25-41.

FERRERES, Lambert (1992A). "A propos d'inanimare et d'interceptus", Museum Helveticum, 49/3, 1992, p. 206.

FERRERES, Lambert (1992B). "La relación entre el ms. Genovefensis 1351 y el Vaticanus Reginensis Lat. 133", Revista Catalana de Teologia 17, 1992, p. 193-199.

FERRERES, Lambert (1992C). "Reminiscencias luciferianas en el Commonitorium de Vicente de Lerins", Anuari de Filologia 15/3, 1992, p. 19-24.

FERRERES, Lambert (1992D). “Tulliana: Catil. 4,18 et 4,20”, Revue des Études Latines 70, 1992, p. 35-36.

FERRERES, Lambert (1992E). “Un par de pasajes ciceronianos en Lucifer de Cálaris”, Faventia 14/2, 1992, p. 99-102.

FERRERES, Lambert (1994A). "Las citas agustinianas en el corpus de Elipando", Charisteria Augustiniana I. Oroz Reta dicata, vol. II, Madrid 1994, p. 165-178.

FERRERES, Lambert (1994B). “Quintiliano y el texto del 'De oratore”, Actas del VIII Congreso Español de Estudios Clásicos, vol. II, Madrid 1994, p. 631-637.

FERRERES, Lambert (1995). “Deux réminiscences des Catilinaires chez Saint Jerôme et Saint Augustin", Faventia 17/1, 1995, p. 119-120.

FERRERES, Lambert (1996): “C. Servilius Ahala: un praenomen incierto”, De Roma al Siglo XX. A.M. Aldama, ed., vol. I, Madrid, UNED, 1996, p. 249-254

FERRERES, Lambert (1999A), Index verborum et locutionum quae in Ps-Cypriani de laude martyrii opusculo inveniuntur, Barcelona, Promociones y Publicaciones Universitarias (PPU), S.A. 
FERRERES, Lambert (1999B). “Fuentes clásicas en el Pseudo-Cipriano De laude martyrii", en A.M. AlDAMA, ed., La Filología Latina hoy. Actualización y perspectivas, vol. I, Madrid 1999, p. 137-142.

FERRERES, Lambert (1999C). “La tradición indirecta en dos restituciones al texto del Pseudo-Cipriano De laude martyrii”, Emérita 57/2, 1999, p. 289-294.

FERRERES, Lambert (2001): “Osservazioni sulle completive in Lucifero da Cagliari”, a LACONI (2001A), p. 145-151.

FERRERES, Lambert (2004A). “La Bible de Pacien”, Pacien de Barcelone el l'Hispanie au IV siècle, París 2004, p. 163-171,

FERRERES, Lambert (2004B), “Introduzione”, a Sonia LACONI, Costanzo II. Ritratto di un imperatore eretico, Roma 2004,

FERRERES, Lambert (2008): “Virgilio Bejarano Sánchez (1922-2007): In memoriam", Cuadernos de Filología Clásica. Estudios Latinos 28, 1, 2008, 167-169.

FERRERES, Lambert (2011A). “Virgilio Bejarano, profesor y maestro”, a MARTíNEZ GÁZQUeZ, DE la CRUZ PALMA, FERRERO HERNÁNDEZ (2011), p. 1097-1099.

FERRERES, Lambert (2011B). “Virgilio Bejarano Sánchez (1922-2007)”, a TUDELA, Montserrat, IZQUIERDO, Pere, eds., La nissaga catalana del món clàssic, Tarragona, Auriga, 2011, p. 461-462.

FERRERES, Lambert (2012). "Texto y tradición textual en el De laude martyrii", en Lessico, argomentazioni e strutture retoriche nella polemica di età cristiana (III-IV sec.), a cura di A. Cappone, Turnout, Brepols, 2012, [Recherches sur les rhétoriques religieuses, 16], 2012, p. 215-221.

FERRERES, Lambert (2014A). “À propos du substantif arentia", Museum Helveticum 71/2, 2014, p. 204-205.

FERRERES, Lambert (2014B). "Acerca del texto de una versión latina del pseudoBasilio Lógos perì askéseos", en Ágalma. Ofrenda desde la Filología Clásica a M. García Teijeiro, A. Martínez Fernández, B. Ortega Villaro, H., Velasco López, H. Zamora Salamanca, eds., Valladolid, Universidad de Valladolid, 2014, p. 1081-1085.

FERRERES, Lambert (2014C). "Beda, Historia Ecclesiastica V XII 1-8, en un manuscrito de la Universidad de Barcelona", Fortunatae. Revista Canaria de Filología, Culturas y Humanidades Clásicas 25, 2014, p. 139-146.

FERRERES, Lambert (2015A). "Hostis = exercitus: Glossa in Apocalypsin Cantabrigiense 1, 177. ¿Un indicio sobre el origen del autor?", Anuari de Filologia. Antiqua et Mediaevalia 4, 2014, 67-69.

FERRERES, Lambert (2015B). “Roger GRYSON (ed.), Incerti auctoris Glossa in Apocalypsin e codice bibliothecae Vniversitatis Cantabrigiensis Dd. X. 16", Anuario de Estudios Medievales, 2015, vol. 45/1, 563-568 (recensió crítica de GRYSON 2013).

Ferreres, Lambert (2016). "Pádraig P. Ó NéIll (ed.), Psalterium Suthantoniense", Anuario de Estudios Medievales 46/1, 2016, 528-533 (recensió crítica).

FERRERES, Lambert (2018A). “El sintagma prepositivo in suspicionem en la lengua de Nepote", Anuari de Filologia. Antiqua et Mediaevalia 8, 2018, p. 329-334.

FERRERES, Lambert (2018B). “Esperança BORRELL VIDAL, Óscar DE LA CRUZ PALMA (eds.), Omnia mutantur: canvi, transformació i pervivència en la cultura clàssica, en les seves llengues i en els seu llegat (II), Barcelona, Edicions de la Universitat de Barcelona, 2016", Anuario de Estudios Medievales 48, 2018, 974-977 (recensió crítica). 
FERRERES, Lambert (2019A): "Sobre el uso de decanto como deponente", Liburna 14, 2019, 241-243.

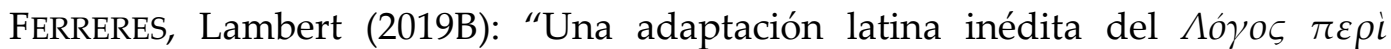

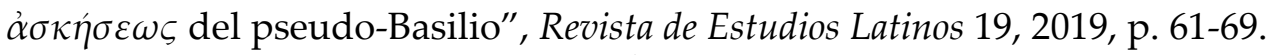

FERRERES, Lambert (2019C): “À propos de 'praesentia' aus sens de $\pi \alpha \rho o v \sigma i \alpha$ ", Museum Helveticum 76/2, 2019, 255-257.

GRYSON, R. (2013), Incerti auctoris Glossa in Apocalypsin e codice bibliothecae Vniversitatis Cantabrigiensis Dd. X. 16 cura et studio R. Gryson, Turnhout, Brepols (CCL 108 $\mathrm{G})$.

LACONI, Sonia (1992). "Virtus cristiana e martyrium nelle pagine del Moriundum esse pro Dei Filio di Lucifero di Cagliari", Sardinia antiqua. Studi in onore di Piero Meloni in occasione del suo settantesimo compleanno , Cagliari 1992, 465-475.

LACONI, Sonia (1997): “Osservazioni di critica testuale sul Moriundum esse pro Dei filio", Annali della Facoltà di Filosofia e Lettere dell'Università degli Studi di Cagliari,

LACONI, Sonia (1998). Luciferi Calaritani. Moriundum esse pro Dei filio, Roma 1998.

LACONI, Sonia (2001A): La figura e l'opera di Lucifero di Cagliari. Una rivisitazione. Atti del I Convegno Internazionale. Cagliari, 5-7 dicembre 1996, Roma 2001, p. 145-151.

LACONI, Sonia (2001B). "Il ritratto di Costanzo II nelle pagine di Lucifero di Cagliari", a LACONI (2001A), 29-62.

LACONI, Sonia (2002). "Figure retoriche e argomentaizone nell'opera di Lucifero di Caglari", Annali della Facolta di Lettere e Filosofia dell'Università di Cagliari, n.s. 20, 2002, 223-299.

MAYER, Marc (2011). “Don Virgilio Bejarano”, a MARTínez GAZQUEZ, DE LA CRUZ PALMA, FERRERO HERNÁNDEZ (2011), p. 1101-1104.

Martínez GÁzQuez, José, de la CRUz PAlma, Óscar, Ferrero Hernández, Cándida (2011). Estudios de Latín Medieval Hispánico. Actas del V Congreso Hispánico de Latín Medieval (Barcelona, 7-10 de septiembre de 2009), Firenze, Sismel - Edizioni del Galluzzo, 2011.

QUETGLAS, Pere (1985). Elementos básicos de filología y lingüística latinas, Barcelona, Editorial Teide, 1985.

UGENTI, Valerio (1980). Luciferi Calaritani De regibus apostaticis et Moriundum esse pro Dei Filio edidit, Italice vertit, testimoniis indicibusque auxit Valerius Ugenti, Lupiis, in aedibus Milellae, 1980 (Studi e testi latini e greci, 1). 